\title{
Bioactive constituents isolated from Atractylodes lancea (Thunb.) DC. rhizome exhibit synergistic effect against cholangiocarcinoma cell
}

This article was published in the following Dove Press journal: Journal of Experimental Pharmacology

\section{Pongsakorn Martviset ${ }^{1,2}$ \\ Wanna Chaijaroenkul',3 \\ Phunuch Muhamad ${ }^{1,4}$ \\ Kesara Na-Bangchang ${ }^{1,3,5}$}

'Center of Excellence in Molecular Biology and Pharmacology of Malaria and Cholangiocarcinoma, Chulabhorn International College of Medicine, Thammasat University, Pathumthani, Thailand; ${ }^{2}$ School of Preclinic, Institute of Science, Suranaree University of Technology, Nakhon Ratchasima, Thailand; ${ }^{3}$ Graduate Program in Bioclinical Sciences, Chulabhorn International College of Medicine, Thammasat University, Pathumthani, Thailand; ${ }^{4}$ Drug Discovery and Development Center, Thammasat University, Pathumthani, Thailand; ${ }^{5}$ WHO-TDR Clinical Coordination and Training Center in Asia and Western Pacific, Pathumthani, Thailand
Correspondence: Kesara Na-Bangchang Center of Excellence in Molecular Biology and Pharmacology of Malaria and Cholangiocarcinoma, Chulabhorn International College of Medicine, Thammasat University, Pathumthani 12121, Thailand

Tel +6625644 44079

Fax +66 25644398

Email kesaratmu@yahoo.com
Background: Cholangiocarcinoma (CCA) is the primary type of bile duct cancer with high morbidity and mortality, particularly in patients with advanced-stage disease. Treatment of CCA remains unsatisfactory due to the lack of sensitive and specific diagnostic tool for early detection as well as effective chemotherapeutics.

Purpose: To investigate cytotoxic interactions between the three major constituents of the rhizomes of Atractylodes lancea (Thunb.) DC., ie, $\beta$-eudesmol (BE), atractylodin (AT), and hinesol (HS), against CCA cell line.

Methods: Cytotoxic activities against the human CCA cells CL- 6 of the dual (BE:AT, BE:HS, and AT:HS) and triple (BE:AT:HS) combinations were evaluated using MTT assay. The cytotoxic interaction of each dual combination was assessed at five concentration ratios (10:0, 7:3, 5:5, $3: 7$, and $0: 10$ ) using isobologram analysis. For triple combination, the concentration ratio used in the experiment was 1:1.5:2.5 (BE:AT:HS) and analysis of the interaction was performed using polygonogram analysis at the concentrations that inhibit cell growth by $50 \%$ and $90 \%$, respectively.

Results: The BE:AT combination produced the additive effect with sum fractional inhibitory concentration of $0.967 \pm 0.02$ (mean \pm SD). The BE:HS and AT:HS combinations produced a synergistic effect with sum fractional inhibitory concentrations of $0.685 \pm 0.08$ and $0.767 \pm 0.09$, respectively. The mixture of the three compounds produced synergistic interaction with combination index values of $0.519 \pm 0.10$ and $0.65 \pm 0.17$ (mean \pm SD) at the concentrations that inhibit cell growth at the $50 \%$ and $90 \%$ leveled, respectively.

Conclusion: Results obtained would guide further development of Atractylodes lancea (Thunb.) DC. as potential anti-CCA chemotherapeutics concerning the appropriate pharmaceutical dosage form.

Keywords: Atractylodes lancea, $\beta$-eudesmol, atractylodin, hinesol, cholangiocarcinoma, cytotoxicity

\section{Introduction}

Cholangiocarcinoma (CCA) is cancer that arises within the bile duct both inside and outside the liver. The cholangiocytes can be transformed into cancer cells by direct activation from carcinogens, or metastasis of other cancers, particularly hepatocellular carcinoma. ${ }^{1,2}$ It is one of the most challenging types of cancer due to the lack of tools for early diagnosis as well as effective chemotherapeutics. The significant risk factor for CCA in People's Republic of China, Korea, Japan, and Southeast Asian countries including Thailand is the consumption of improperly cooked cyprinoid fish which contains infective metacercaria of the liver fluke Opisthorchis viverrini, O. felines, 
and Clonorchis sinensis, together with dimethylnitrosamine (DMN) from fermented meat. ${ }^{3-5}$ In Thailand, CCA shared approximately $11 \%$ of the cases among new cancer cases reported in 2015, with the highest incidence in the Northeastern region (about $60 \%$ of all cases). ${ }^{6}$ The efficacy of existing chemotherapy and radiotherapy are limited only to patients with early-stage disease. ${ }^{7,8}$ Treatment efficacy of the standard drugs 5-fluorouracil (5-FU), gemcitabine, or combinations of these drugs with cisplatin is unsatisfactory with low survival rate and high metastatic rate. ${ }^{9}$ Less than $5 \%$ of the advanced-stage patients survive for up to 5 years. ${ }^{10,11}$ Research on discovery and development of new effective alternative drugs is urgently required to control this type of cancer.

Numerous studies have been carried out in efforts to discover effective cancer chemotherapeutic agents from plant sources with low toxicity. Histological evidence support the role of plants as promising sources of anticancer drugs, ie, vincristine, vinblastine, etoposide, teniposide, paclitaxel, vinorelbine, docetaxel, topotecan, and irinotecan. ${ }^{12}$ With regard to $\mathrm{CCA}$, a number of plants and plant-derived compounds have been investigated for their anti-CCA potentials both in vitro and in animal models. These include triptolide from Tripterygium wilfordii, ${ }^{13}$ ubiquitous tannic acid, ${ }^{14}$ and Atractylodes lancea (Thunb.) DC. (AL) and its isolated compounds ( $\beta$-eudesmol [BE], atractylodin [AT], atractylon, hinesol [HS], etc). ${ }^{15-19}$ The interest of our research group has been focusing on research and development of AL as a potential chemotherapeutic for CCA. AL is the therapeutic plant growing in tropical and subtropical zones of Asia such as People's Republic of China, Japan, and Thailand. Its dried rhizome is commonly used in Chinese ("Cang Zhu"), Japanese campo ("So-jutsu”), as well as Thai ("Khod-Kha-Mao") traditional medicines. In Chinese traditional medicine, AL has been used as an important crude drug for treatment of rheumatic diseases, digestive disorders, night blindness, and influenza. ${ }^{20}$ The pharmacological activities of AL rhizomes have previously been reviewed. ${ }^{21}$ Phytochemical investigations have revealed a series of sesquiterpenoids, monoterpenes, polyacetylenes, phenolic acids, and steroids from AL rhizomes. ${ }^{22-29}$ The major constituents are AT (14\%), BE $(6 \%)$, atractylon $(2 \%)$, and $\mathrm{HE}(1 \%)$. Results from a series of our study confirm anti-CCA potential and safety profiles of both the crude ethanolic extract and the purified compound BE both in vitro and in animal models. ${ }^{16-19}$ The present study aimed to investigate the cytotoxic interactions on human CCA between the three major isolated compounds from the AL rhizome, ie, BE, AT, and HS.

\section{Methods \\ Cell culture and test compounds}

The CCA cell line CL-6 was kindly provided by Associate Professor Dr Adisak Wongkajornsilp, Department of Pharmacology, Faculty of Medicine (Siriraj Hospital), Mahidol University. The experimental use of the CL-6 cell was approved by Thammasat University Biosafety Committee (008/2561). The cell was cultured in Roswell Park Memorial Institute 1640 medium (Gibco Co. Ltd., Grand Island, NY, USA). The culture medium was supplemented with $10 \%(\mathrm{v} / \mathrm{v})$ heated fetal bovine serum and $100 \mathrm{IU} / \mathrm{mL}$ of antibiotic-antimycotic solution (Gibco Co. Ltd.). All cells were maintained at $37^{\circ} \mathrm{C}$ in 5\% $\mathrm{CO}_{2}$ atmosphere and $95 \%$ humidity (HERACELL 150i, Thermo Scientific, Waltham, MA, USA).

The bioactive constituents of AL rhizome under investigation, ie, BE, AT, HS, and 5-FU were purchased from Wako (Wako Ltd., Osaka, Japan). The stock solution of each compound was prepared in 50\% ethanol at the concentration of $5 \mathrm{mg} / \mathrm{mL}$, and the working solution was prepared freshly before use.

\section{Cytotoxic assay}

The cytotoxic effects of dual and triple combinations of BE, AT, and HS on CL-6 were evaluated using MTT assay (Sigma-Aldrich, St. Louis, MO, USA) according to the manufacturer's protocol. The cells $\left(0.75 \times 10^{4}\right.$ cells/well $)$ were seeded into each well of a 96-well microtiter plate and incubated at $37^{\circ} \mathrm{C}$ under $5 \% \mathrm{CO}_{2}$ atmosphere for 24 hours. Cell confluence was examined under the light microscope.

\section{Analysis of the cytotoxic activity of dual combinations}

The cytotoxic interaction of each dual combination, ie, BE-AT, BE-HS, or AT-HS was evaluated at the five concentration ratios $(10: 0,7: 3,5: 5,3: 7$, and $0: 10)$, with a serial dilution for each combination pair. The highest concentration used was $200 \mu \mathrm{g} / \mathrm{mL}(899 \mu \mathrm{M} \mathrm{BE}, 1098 \mu \mathrm{M} \mathrm{AT}$, and $899 \mu \mathrm{M}$ HS). Following incubation at $37^{\circ} \mathrm{C}$ for 48 hours, $20 \mu \mathrm{L}$ of the MTT reagent was added into each well and further incubated for an additional 4 hours. The cell suspension was carefully removed, and dimethyl sulfoxide (Ameresco, Solon, $\mathrm{OH}$, USA) was added, and the absorbance was measured at 565 $\mathrm{nm}$ within 15 minutes. 5-FU was used as a reference compound for comparison of cytotoxic activity. The experiment was performed at least three times, each time in triplicate. The concentration that inhibits cell growth by $50 \%\left(\mathrm{IC}_{50}\right)$ of each compound (from the starting ratios of 10:0 and 0:10) and was estimated using Calcusyn ${ }^{\mathrm{TM}}$ (Biosoft, Cambridge, 
UK). The fractional inhibitory concentration (FIC) index of each combination pair (representing combination scores) and the sum FIC of five distinctive ratios was calculated as the ratio of $\mathrm{IC}_{50}$ of the combination and that of each compound alone. The isobologram of each combination interaction was generated from the average sum FIC index (sum of the $\mathrm{IC}_{50}$ of each combination pair divided by $\mathrm{IC}_{50}$ of each single compound). Sum FIC values indicate the types of cytotoxic interactions: synergism if sum FIC $<1$, addition if sum FIC $=1$, and antagonism if sum FIC $>1$.

\section{Analysis of the cytotoxic activity of triple combinations}

The concentration ratio used for evaluation of cytotoxic activity of the triple combination (BE:AT:HS) was based on the previously reported average $\mathrm{IC}_{50}$ value of each compound from our laboratory $(20.1,31.0$, and $52.3 \mu \mathrm{M}$ for BE, AT and HS, respectively). ${ }^{15,16}$ The estimated combination ratio of BE:AT:HS used in the experiment was $1: 1.5: 2.5$. The experiment was performed as previously described for the dual combinations (three independent experiments, triplicate each). The combination index (CI) value at the $\mathrm{IC}_{50}$ or $\mathrm{IC}_{90}$ was determined as follow: $\left(\mathrm{IC}_{50}\left[\right.\right.$ or $\left.\mathrm{IC}_{90}\right]$ of $\mathrm{BE}$ in the triple combination divided by $\mathrm{IC}_{50}\left[\right.$ or $\left.\mathrm{IC}_{90}\right]$ of $\mathrm{BE}$ alone $)+\left(\mathrm{IC}_{50}\right.$ [or $\mathrm{IC}_{90}$ ] of $\mathrm{AT}$ in the triple combination divided by $\mathrm{IC}_{50}$ [or $\left.\mathrm{IC}_{90}\right]$ of AT alone $)+\left(\mathrm{IC}_{50}\left[\right.\right.$ or $\left.\mathrm{IC}_{90}\right]$ of $\mathrm{HS}$ in the triple combination divided by $\mathrm{IC}_{50}$ [or $\mathrm{IC}_{90}$ ] of compound $\mathrm{HS}$ alone). For analysis of combination interaction, the polygonogram was generated using CompuSyn ${ }^{\mathrm{TM}}$ (ComboSyn Inc., Paramus, NJ, USA). Criteria for classification of the types of cytotoxic interaction are as follows: very strong synergism if $\mathrm{CI}<0.1$, strong synergism if $\mathrm{CI}=0.1-0.3$, synergism if $\mathrm{CI}$ $=0.3-0.7$, moderate synergism if $\mathrm{CI}=0.7-0.85$, slight synergism if $\mathrm{CI}=0.85-0.90$, nearly additive if $\mathrm{CI}=0.9-1.10$, slight antagonism if $\mathrm{CI}=1.10-1.20$, moderate antagonism if $\mathrm{CI}$ $=1.20-1.45$, antagonism if $\mathrm{CI}=1.45-3.3$, strong antagonism if $\mathrm{CI}=3.3-10$, and very strong antagonism if $\mathrm{CI}>10 .{ }^{30,31}$

\section{Results}

The $\mathrm{IC}_{50}$ values (mean $\pm \mathrm{SD}$ ) of $\mathrm{BE}$, AT, HS and 5-FU were $21.5 \pm 2.12,24 \pm 1.98,91.81 \pm 8.0$, and $122 \pm 2.08 \mu \mathrm{M}$, respectively. For dual combinations, the sum FIC indexes (mean $\pm \mathrm{SD}$ ) indicative of types of drug interaction were

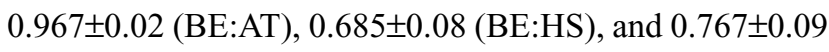
(AT:HS), suggesting additive, synergistic, and synergistic interaction, respectively. The $\mathrm{IC}_{50}$ of $\mathrm{BE}, \mathrm{AT}$, and $\mathrm{HS}$ alone were $21.5 \pm 2.12,24 \pm 1.98$, and $91.81 \pm 8.0 \mu \mathrm{M}$, respectively. The $\mathrm{IC}_{50}$ of the reference drug $5-\mathrm{FU}$ was $90 \pm 7.9 \mu \mathrm{M}$. The isobolograms of FICs of the three dual combinations are illustrated in (Figure 1). For triple combinations, the CI (mean $\pm \mathrm{SD}$ ) values at the $\mathrm{IC}_{50}$ and $\mathrm{IC}_{90}$ levels were $0.519 \pm 0.10$ and $0.65 \pm 0.17$, indicating synergistic and moderately synergistic interaction, respectively (Figure 2).

\section{Discussion}

Traditional herbal products are heterogeneous in nature. It is likely that the existence of various components from the plant parts used could act synergistically or at least additively and provide therapeutic advantages over the use of a single active constituent isolated from the plant with regard to both clinical efficacy and tolerability. This supports the traditional uses of herbal medicines as concoction for most diseases or pathological conditions. The study was the first that confirmed the cytotoxic synergistic interaction of the three major compounds from AL rhizome, ie, $\beta$-eudesmol (BE), atractylodin (AT), and hinesol (HS), on the human CCA cell line CL-6. Based on the $\mathrm{IC}_{50}$ value, $\mathrm{BE}$ or AT alone exerted its cytotoxic potency of about four times that of the reference drug 5-FU, while the potency of cytotoxic activity of HS alone was similar to 5-FU. Interestingly, the dual and triple combinations containing the HS component (BE:HS, AT:HS, BE:AT:HS) produced synergistic interaction. On the other hand, the BE:AT combination produced only additive interaction despite relatively higher amounts of both compounds compared to BE:HS or AT:HS combination ( $6 \%, 14 \%$, and $1 \%$ for BE, AT and HS, respectively), and in addition, relatively weak cytotoxic activity of $\mathrm{HS}\left(\mathrm{IC}_{50}\right.$ : 21.5, 24.0 and $91.8 \mu \mathrm{M}$ for BE, AT, and HS, respectively). Results of this in vitro study confirmed that of the previous in vivo studies in the CCA-xenografted nude mouse model which demonstrated potent activity of the AL extract and BE at tolerable dose levels. ${ }^{16-18}$ It is noted however that, with the relatively low amounts of all the three constituents in the AL extract, its anti-CCA activity observed in the in vivo CCAxenografted mouse and $O$. viverrini/dimethylnitrosamineinduced CCA hamster models was shown to be higher than the isolated compound BE alone. ${ }^{17-19}$ Significant inhibitory effects of AL extract on CCA tumor growth ( $2 \%$ of untreated control) were observed at the dose levels of 1,000, 3,000 and $5,000 \mathrm{mg} / \mathrm{kg}$ body weight, while the inhibitory effect of only $70 \%$ was found in the 5 -FU treated mice. ${ }^{17}$ The survival time of mice was about 3-4 times more prolonged following all dose levels of AL extract compared with 5-FU and untreated control. More importantly, the extract also produced potent inhibitory effects on lung metastasis. The AL extract at 5,000,3,000, 1,000 mg/kg body weight and 
A

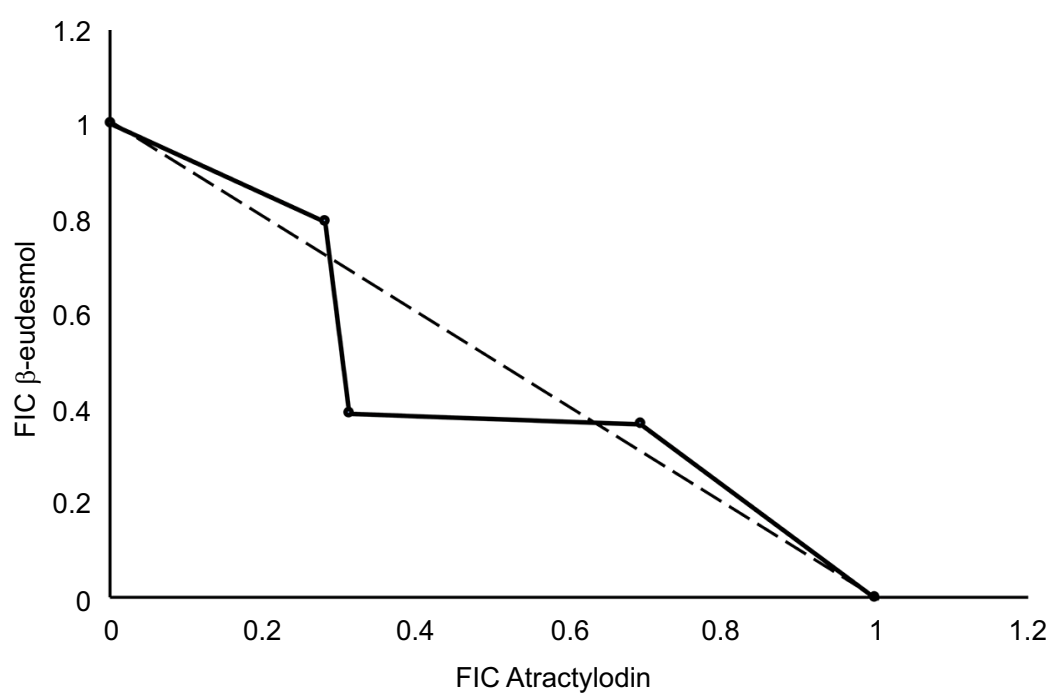

B

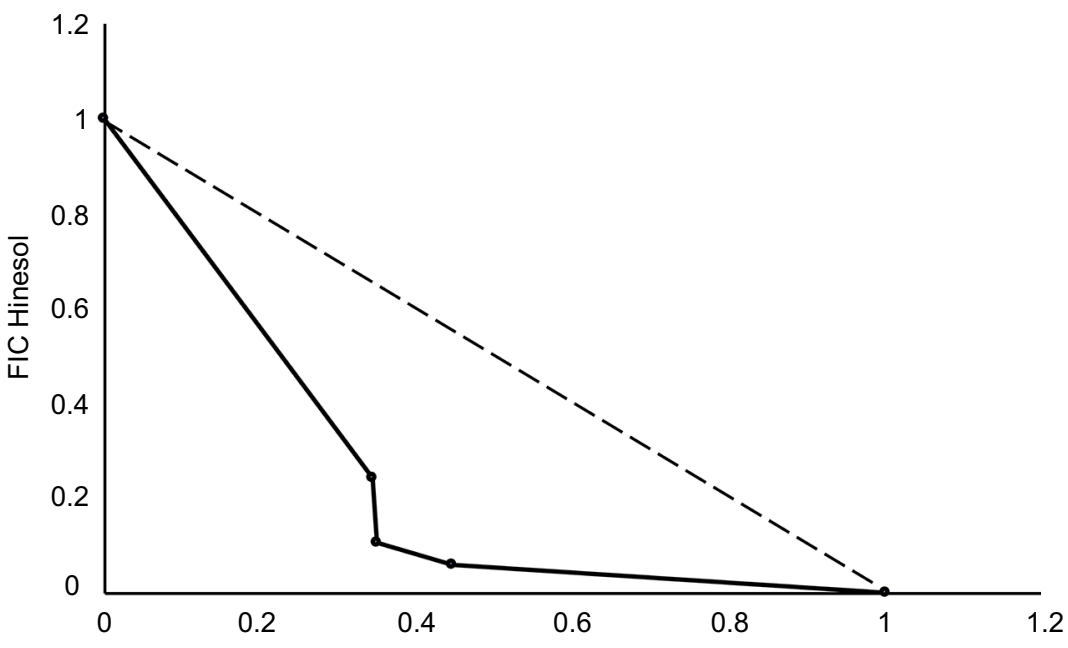

C

FIC Atractylodin

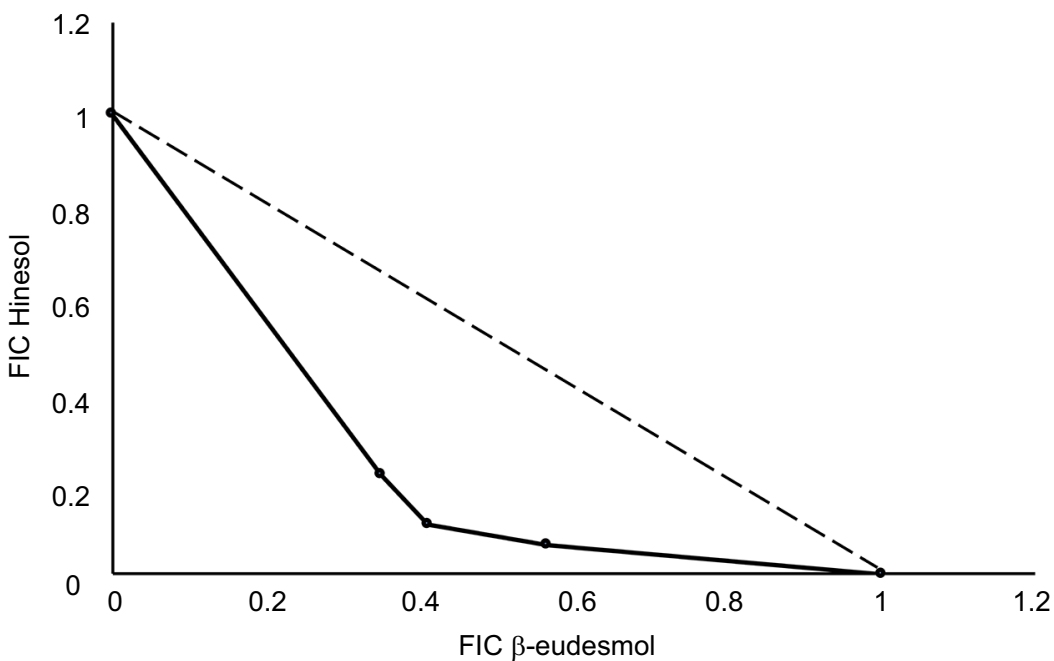

Figure I Isobolograms of the sum FIC values (mean \pm SD) of the dual combinations of the three compounds isolated from AL and types of interactions: (A) BE and AT combination (additive effect with sum FIC of $0.967 \pm 0.02$ ); (B) AT and HS (synergistic effect with sum FIC of $0.685 \pm 0.08$ ); and (C) BE and HS (synergistic effect with sum $\mathrm{FIC}$ of $0.767 \pm 0.09)$.

Abbreviations: AL, Atractylodes lancea (Thunb.) DC.; AT, atractylodin; BE, b-eudesmol; FIC, fractional inhibitory concentration; HS, hinesol. 


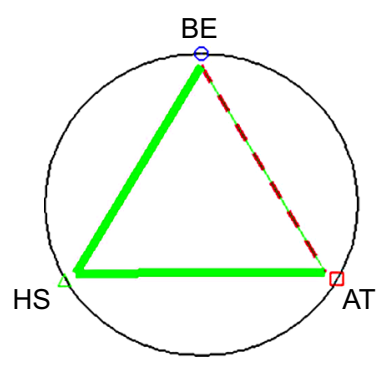

A

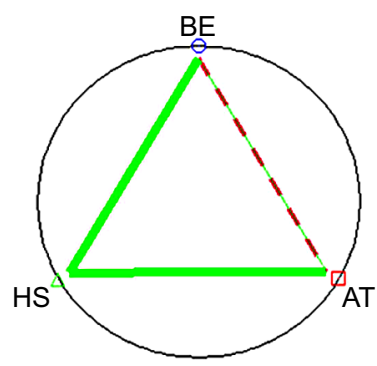

B
Figure 2 Polygonograms of the sum $\mathrm{Cl}$ values (mean $\pm \mathrm{SD}$ ) of the triple combinations of BE:AT:HS (concentration ratio $=\mathrm{I}: \mathrm{I} .5: 2.5)$ at $(\mathbf{A}) I \mathrm{C}_{50}$ and $\mathbf{( B )} I \mathrm{CC}_{90}$. The solid lines represent synergism; dashed lines represent nearly additive effect).

Abbreviations: $\mathrm{AT}$, atractylodin; $\mathrm{BE}$, b-eudesmol; $\mathrm{Cl}$, combination index; $\mathrm{HS}$, hinesol; IC50, concentration that inhibits cell growth by $50 \%$; IC 90 , concentration that inhibits cell growth by $90 \%$.

5-FU and untreated control, respectively, resulted in 95\%, $75 \%, 30 \%, 50 \%$, and $10 \%$ inhibition of lung metastasis. Comparable anti-CCA activity and inhibitory activity on lung metastasis were also observed with BE. ${ }^{19}$ In the hamster model, the promising anti-CCA activity of AL was observed at all dose levels, particularly at the highest oral dose level of $5,000 \mathrm{mg} / \mathrm{kg}$ body weight for 30 days. The median survival time was significantly prolonged (about two times) in mice treated with the extract at all dose levels compared with 5-FU treated and untreated control groups. ${ }^{19}$ Altogether, results suggest that the three major compounds in the $\mathrm{AL}$ rhizomes play an important role in the anti-CCA activity of AL. The study supports synergistic interaction of the active ingredients observed in all animal models. ${ }^{16-19}$ Other constituents including atractylon ( $2 \%$ yield) as well as other constituents may also interact additively or synergistically with these compounds to exert promising anti-CCA activity of the AL extract. It is noted however that results of the in vitro and in vivo studies, particularly with different CCA cell types in various CCA cells, may be different due to complex environment. CCA encompasses at least three pathological conditions, ie, intrahepatic, hilar, and distal CCA, which respond differently to chemotherapeutic drugs. The CL-6 cell line used in the study was obtained from patients with intrahepatic CCA, which is the most common CCA type in Southeast Asia. Nevertheless, studies in other types of CCA are encouraged for further development of AL for CCA. The potential anticancer and antiangiogenesis properties of AL extract and these major constituents, particularly BE, have been well demonstrated in various types of animal and human cancers, these include murine blastoma cells, HeLa (human cervical cells), SGC-7901 (human gastric cancer cells), BEL-7402 (human liver cancer cells), H33, S180,
HL-60 leukemic cells, and gastric cancer. ${ }^{32-36}$ For CCA, the cytotoxic activity, anticlonogenic activity, antioxidative activity, and inhibitory activity on cell invasion and angiogenesis of the crude ethanolic extract of AL have previously been demonstrated by our group. ${ }^{16}$

Oral pharmaceutical formulation of the standardized crude extract rather than each isolated compound should be considered for further development of AL as promising antiCCA chemotherapeutics. The multi-ingredient characteristics of the plant extract would be expected to optimize therapy regarding both efficacy (synergistic anti-CCA activity) and tolerability (buffering effect). Apart from therapeutic benefit, this approach offers an advantage over the isolated compounds concerning both time consumption and resource consumption during the development process. The strategy of drug development based on multiple targets of action using systems pharmacology, rather than a single molecular target, would be a more suitable approach for investigation of mechanisms of action of herbal medicines.

\section{Conclusion}

The results obtained would guide further development of Atractylodes lancea (Thumb.) DC. as potential anti-CCA chemotherapeutics concerning the appropriate pharmaceutical dosage form.

\section{Acknowledgment}

The study was supported by Thammasat University, Center of Excellence in Pharmacology and Molecular Biology of Malaria and Cholangiocarcinoma of Thammasat University; National Research Council of Thailand (NRCT) and National Research University Project of Thailand (NRU); and the Office of Higher Education Commission of Thailand.

\section{Disclosure}

The authors report no conflicts of interest in this work.

\section{References}

1. Renshaw K. Malignant neoplasms of the extrahepatic biliary ducts. Ann Surg. 1922;76(2):205-221.

2. Lazaridis KN, Gores GJ. Cholangiocarcinoma. Gastroenterology 2005;128(6):1655-1667.

3. Sripa B, Kaewkes S, Sithithaworn P, et al. Liver fluke induces cholangiocarcinoma. PLoS Med. 2007;4(7):e201.

4. Leyva-Illades D, Mcmillin M, Quinn M, Demorrow S. Cholangiocarcinoma pathogenesis: Role of the tumor microenvironment. Transl Gastrointest Cancer. 2012;1(1):71-80.

5. Rizvi S, Gores GJ. Molecular Pathogenesis of Cholangiocarcinoma. Dig Dis. 2014;32(5):564-569.

6. National Cancer Institute of Thailand. Hospital-based cancer registry annual report. Bangkok: National Cancer Institute of Thailand. 2015.

7. Nakeeb A, Pitt HA. Radiation therapy, chemotherapy and chemoradiation in hilar cholangiocarcinoma. HPB. 2005;7(4):278-282. 
8. Ramírez-Merino N, Aix SP, Cortés-Funes H. Chemotherapy for cholangiocarcinoma: An update. World J Gastrointest Oncol. 2013;5(7):171-176.

9. Bridgewater J, Galle PR, Khan SA, et al. Guidelines for the diagnosis and management of intrahepatic cholangiocarcinoma. J Hepatol. 2014;60(6):1268-1289.

10. Ciombor KK, Goff LW. Advances in the management of biliary tract cancers. Clin Adv Hematol Oncol. 2013;11(1):28-34.

11. Rogers JE, Law L, Nguyen VD, et al. Second-line systemic treatment for advanced cholangiocarcinoma. J Gastrointest Oncol. 2014;5(6): $371-413$.

12. Dholwani KK, Saluja AK, Gupta AR, Shah DR. A review on plantderived natural products and their analogs with anti-tumor activity. Indian J Pharmacol. 2008;40(2):49-58.

13. Tengchaisri T, Chawengkirttikul R, Rachaphaew N, Reutrakul V, Sangsuwan R, Sirisinha S. Antitumor activity of triptolide against cholangiocarcinoma growth in vitro and in hamsters. Cancer Lett. 1998;133(2):169-175.

14. Naus PJ, Henson R, Bleeker G, Wehbe H, Meng F, Patel T. Tannic acid synergizes the cytotoxicity of chemotherapeutic drugs in human cholangiocarcinoma by modulating drug efflux pathways. $J$ Hepatol. 2007;46(2):222-229.

15. Mahavorasirikul W, Viyanant V, Chaijaroenkul W, Itharat A, NaBangchang K. Cytotoxic activity of Thai medicinal plants against human cholangiocarcinoma, laryngeal and hepatocarcinoma cells in vitro. BMC Complement Altern Med. 2010;10:55.

16. Plengsuriyakarn T, Viyanant V, Eursitthichai V, et al. Cytotoxicity, toxicity, and anticancer activity of Zingiber officinale Roscoe against cholangiocarcinoma. Asian Pac J Cancer Prev. 2012;13(9):4597-4606.

17. Plengsuriyakarn T, Viyanant V, Eursitthichai V, et al. Anticancer activities against cholangiocarcinoma, toxicity and pharmacological activities of Thai medicinal plants in animal models. BMC Complement Altern Med. 2012;12:23

18. Plengsuriyakarn T, Karbwang J, Na-Bangchang K. Anticancer activity using positron emission tomography-computed tomography and pharmacokinetics of $\beta$-eudesmol in human cholangiocarcinoma xenografted nude mouse model. Clin Exp Pharmacol Physiol. 2015;42(3):293-304.

19. Plengsuriyakarn T, Matsuda N, Karbwang J, Viyanant V, Hirayama K, Na-Bangchang K. Anticancer Activity of Atractylodes lancea (Thunb.) DC in a Hamster Model and Application of PET-CT for Early Detection and Monitoring Progression of Cholangiocarcinoma. Asian Pacific Journal of Cancer Prevention. 2015;16(15):6279-6284.

20. Ouyang Z, Zhang L, Zhao M, Wang P, Wei Y, Fang J. Identification and quantification of sesquiterpenes and polyacetylenes in Atractylodes lancea from various geographical origins using GC-MS analysis. Revista Brasileira de Farmacognosia. 2012;22(5):957-963.
21. Na-Bangchang K, Plengsuriyakarn T, Karbwang J. Research and Development of Atractylodes lancea (Thunb) DC. as a Promising Candidate for Cholangiocarcinoma Chemotherapeutics. Evid Based Complement Alternat Med. 2017;2017:5929234:1-16.

22. Chen Z-L, Cao W-Y, Zhou G-X, Wichtl M. A sesquiterpene lactam from Artractylodes macrocephala. Phytochemistry. 1997;45(4):765-767.

23. Kano Y, Komatsu K-Ichi, Saito K-Ichi, Bando H, Sakurai T. A new polyacetylene compound from Atractylodes rhizome. Chem Pharm Bull. 1989;37(1):193-194.

24. Yahara S, Higashi T, Iwaki K, et al. Studies on the constituents of Atractylodes lancea. Chem Pharm Bull. 1989;37(11):2995-3000.

25. Resch M, Steigel A, Chen ZL, Bauer R. 5-Lipoxygenase and cyclooxygenase-1 inhibitory active compounds from Atractylodes lancea. J Nat Prod. 1998;61(3):347-350.

26. Zhang Y, Xu S, Lin Y, Sb X, Lin C. Gastrointestinal inhibitory effects of sesquiterpene lactones from Atractylodes macrocephala. Zhong Yao Cai. 1999;22(12):636-640.

27. Ding H-Y, Wu Y-C, Linc H-C. Phytochemical and Pharmacological Studies on Chinese Changzhu. J Chin Chem Soc. 2000;47(3):561-566.

28. Resch M, Heilmann J, Steigel A, Bauer R. Further phenols and polyacetylenes from the rhizomes of Atractylodes lancea and their antiinflammatory activity. Planta Med. 2001;67(5):437-442.

29. Kitajima J, Kamoshita A, Ishikawa T, et al. Glycosides of Atractylodes lancea. Chem Pharm Bull. 2003;51(6):673-678.

30. Chou TC, Martin N. CompuSyn for Drug Combinations: PC Software and User's Guide: A Computer Program for Quantitation of Synergism and Antagonism in Drug Combinations, and the Determination of IC50 and ED50 and LD50 Values. Paramus, NJ: ComboSyn Inc.; 2005.

31. Chou TC. Theoretical basis, experimental design, and computerized simulation of synergism and antagonism in drug combination studies. Pharmacol Rev. 2006;58(3):621-681.

32. Tsuneki H, Ma EL, Kobayashi S, et al. Antiangiogenic activity of betaeudesmol in vitro and in vivo. Eur J Pharmacol. 2005;512(2-3):105-115.

33. Ma EL, Li YC, Tsuneki H, El M, Yc L, et al. Beta-eudesmol suppresses tumour growth through inhibition of tumour neovascularisation and tumour cell proliferation. JAsian Nat Prod Res. 2008;10(1-2):159-167.

34. Mazzio EA, Soliman KF. In vitro screening of tumoricidal properties of international medicinal herbs: part II. Phytother Res. 2010;24(12):1813-1824.

35. Zhao JH, Sun JX, Gao P, et al. Fast-track surgery versus traditional perioperative care in laparoscopic colorectal cancer surgery: a metaanalysis. BMC Cancer. 2014;14:607.

36. Masuda Y, Kadokura T, Ishii M, Takada K, Kitajima J. Hinesol, a compound isolated from the essential oils of Atractylodes lancea rhizome, inhibits cell growth and induces apoptosis in human leukemia HL-60 cells. J Nat Med. 2015;69(3):332-339.
Journal of Experimental Pharmacology

\section{Publish your work in this journal}

The Journal of Experimental Pharmacology is an international, peerreviewed, open access journal publishing original research, reports, reviews and commentaries on all areas of laboratory and experimental pharmacology. The manuscript management system is completely online and includes a very quick and fair peer-review system.
Dovepress

Visit http://www.dovepress.com/testimonials.php to read real quotes from published authors. 